\title{
A survey of diagnostic ultrasound within the physiotherapy profession for the design of future training tools
}

\author{
Sharmaine McKiernan*, Pauline Chiarelli, Helen Warren-Forward \\ School of Health Sciences, The University of Newcastle, Callaghan, NSW, Australia
}

\section{A R T I C L E I N F O}

\section{Article history:}

Received 21 May 2010

Received in revised form

10 August 2010

Accepted 17 August 2010

Available online 9 September 2010

\section{Keywords:}

Physiotherapy

Biofeedback

Diagnostic ultrasound

Ultrasound training

\begin{abstract}
A B S T R A C T
The expansion of diagnostic ultrasound outside of the traditional radiology profession into the physiotherapy profession is occurring. The purpose of this study was to determine if physiotherapists are purchasing diagnostic ultrasound machines, receiving training in the modality and what imaging procedures they are performing. For the design of future training tools, also investigated were the methods of training that physiotherapists might find most beneficial and what content they deem necessary to be covered during such training for the use of diagnostic ultrasound for their profession.

An e-mail invitation was sent to physiotherapists throughout Australian who were registered on two databases, asking them to complete a web based survey. The survey was comprised of 18 questions including open and closed items. The data was then categorised into themes in accordance with the purpose of the study.

Of the respondents, 39\% did not own a diagnostic ultrasound machine, $33 \%$ had access to a machine that was owned by their employer and $18 \%$ actually owned a machine themselves. Training in diagnostic ultrasound had been received by $61 \%$ of the respondents however for $67 \%$ of those who had been trained, this training had only lasted for several hours, not days or weeks. For future training in ultrasound the majority of respondents would prefer either a workshop or DVD to cover imaging anatomy, the use of machine controls and scanning the pelvic floor, abdominal muscles and shoulder.

From this survey it can be concluded that physiotherapists have an interest in or are using diagnostic ultrasound in their practice. While some form of training is being provided, further training is considered necessary and wanted by the physiotherapists so training tools need to be developed.
\end{abstract}

(C) 2010 The College of Radiographers. Published by Elsevier Ltd. All rights reserved.

\section{Introduction}

Diagnostic ultrasound is traditionally used within the radiology profession as a diagnostic tool; recently it has expanded into other health professions such as physiotherapy. ${ }^{1-4}$ Physiotherapists need training and guidance in this modality from experts in the field, however, in order to maximise the help provided, the experts in the field need an understanding of the physiotherapists' requirements and preference for training methods.

Physiotherapists use diagnostic ultrasound mostly for biofeedback, as a tool in functional retraining of muscles. As a part of the motor re-learning process, a patient learns how to correctly perform a functional task and with time and practice, the aim is that the task should become more automatic and thus should be of benefit to the patient. $^{4-7}$ The visual biofeedback provided by diagnostic ultrasound is used to help both the patient and therapist confirm that

\footnotetext{
* Corresponding author. Tel.: +612 49218861.

E-mail address: sharmaine.mckiernan@newcastle.edu.au (S. McKiernan).
}

a specific functional muscle task has been learnt and is being performed correctly. Diagnostic ultrasound is also an effective assessment tool providing measurements of muscle thickness. ${ }^{4-7}$

Physiotherapists currently use diagnostic ultrasound to provide visual feedback on co-ordinated abdominal muscle activation. They teach patients with lower back pain to perform an abdominal drawing-in manoeuvre to encourage activation of the transversus abdominis muscles and to co-ordinate this with the spinal multifidus muscles to stabilise the trunk and facilitate a decrease in symptoms. ${ }^{6,8,9}$ Visual biofeedback provided by diagnostic ultrasound is also used in the functional retraining of pelvic floor muscles in patients who present with lower urinary tract dysfunction. ${ }^{5,10}$ Patients are taught to perform a pelvic floor muscle contraction which elevates the pelvic floor and lifts the base of the urinary bladder. Such pelvic floor muscle imaging can be undertaken using transperineal and/or transabdominal ultrasound. ${ }^{10}$ Information about the supporting function of the pelvic floor muscles during manoeuvres such as sneezing, coughing and valsalva can also be assessed by imaging the bladder. ${ }^{11,12}$ 
This paper reports on data derived from a survey of physiotherapists and forms part of a larger project that aims to design training tools for physiotherapists in diagnostic ultrasound. This survey was undertaken to determine physiotherapy ownership of diagnostic ultrasound machines and the machine capabilities, including transducer types and Doppler capabilities, the training being received and who is providing this training. The survey also explored what procedures are being performed by physiotherapists clinically. In order to provide optimal professional development for physiotherapists, it is important to have a clear understanding of what methods of training would best suit the physiotherapist and what areas of training they require. If an understanding of the current situation is obtained, any training can then be tailored to the requirements of the physiotherapist to make it beneficial to their profession.

\section{Method}

A survey was designed on Survey Monkey (www.surveymonkey. com, California Office: 640 Oak Grove Avenue, Menlo Park, CA 94025, USA) a web based program. An e-mail inviting participation was sent to physiotherapists registered on two databases of physiotherapists throughout Australia, who had attended workshops, ordered texts or who regularly attend seminars held at the University of Newcastle. Ethics approval for this survey was granted by the Hunter New England Human Research Ethics Committee.

The survey was comprised of 18 items including open and closed questions, see Table 1 . Closed questions sought information related to respondent demographics and diagnostic ultrasound usage. The open questions sought information related to details of their ultrasound machine, training received and perceived outcomes related to their training. The open questions allowed respondents to use their own words thus providing detail indepth. ${ }^{13,14}$

The data was then categorised in accordance with the purpose of the study. Patterns of information were investigated and incidental comments analysed for relevance and importance.

\section{Results}

A total of 99 surveys were completed from an e-mail invitation of 483 , giving a response rate of $20.5 \%$. Respondents' age groups were between less than 30 and greater than 55 years, with the largest respondent group being less than 30 years of age. Respondents were $79 \%$ female and $75 \%$ worked in a private physiotherapy clinic. In terms of location, $61 \%$ worked in metropolitan areas, $23 \%$ regional and $16 \%$ rural.

Respondents reported working in a wide variety of clinical physiotherapy areas as shown in Fig. 1. While the most common clinical physiotherapy practice related to musculoskeletal (MSK), other clinical areas reported by physiotherapists, shown on the graph by other, included paediatrics, lymphoedema, aged care, maternity and the emergency department.

\section{The diagnostic ultrasound machine}

Of the respondents, 39\% reported not owning a diagnostic ultrasound machine and 33\% indicated that the practice they worked for owned a machine. Only $18 \%$ of the respondents actually owned a machine themselves.

When asked the open question on the specific brand of their ultrasound machine, responses varied from "?", "unsure", "looking to" or "in the process of purchasing one", or "some ancient thing". The most popular machine brand listed was Mindray (Address: Mindray Building, Keji 12th Road South, High-tech Industrial Park,
Table 1

The 18 items of the questionnaire.

What is your age group?
Are you male or female?
Do you work in the private or public system?
Do you work in a rural, regional or metropolitan area?
What area/s of physiotherapy do you work in?
Do you own a diagnostic ultrasound machine?
What brand is your diagnostic ultrasound machine?
Does your diagnostic ultrasound machine have
Doppler and colour capabilities?
What transducers do you have?
Have you had any training in diagnostic ultrasound?
If you have received training, how long was this training for?
Who provided this training?
For how long have you been using diagnostic ultrasound?
What diagnostic ultrasound procedures do you perform?
How many times a day on average would you use diagnostic ultrasound?
What methods of training in diagnostic ultrasound would best suit you?
What do you feel you need more information about?
What diagnostic ultrasound procedures would you most like to be trained in?

Nanshan, Shenzhen 518057, P.R. China) with 20 of the respondents indicating they had access to this brand of machine. When asked if their machine had Doppler and colour capabilities, 85\% of respondents skipped the question, $14 \%$ selected Doppler and 5\% selected colour.

The types of transducers respondents had access to, was another open question and there were a few who stated 'unsure' while many wrote about "flat" and "round heads". A $3 \mathrm{MHz}$ curved transducer was the most common response and just over $40 \%$ of respondents stated they had access to both a curved and linear transducer.

\section{Training}

Of the respondents, $61 \%$ had received some form of training in diagnostic ultrasound. For $67 \%$ of these, this training had only lasted for several hours, not days or weeks. In response to the open question about, who had supplied the training, responses included "supplier of machine", "in house", "radiology department", "university lecturers", "gynaecologists" and "specialist physiotherapist".

\section{Use of the modality}

In relation to the use of the diagnostic ultrasound machine, 33\% of respondents had never used a machine in practice and of those that had, $24 \%$ had used it for a period of less than one year. On average, for those respondents using the modality in practice, it was only being used 1-2 times a day.

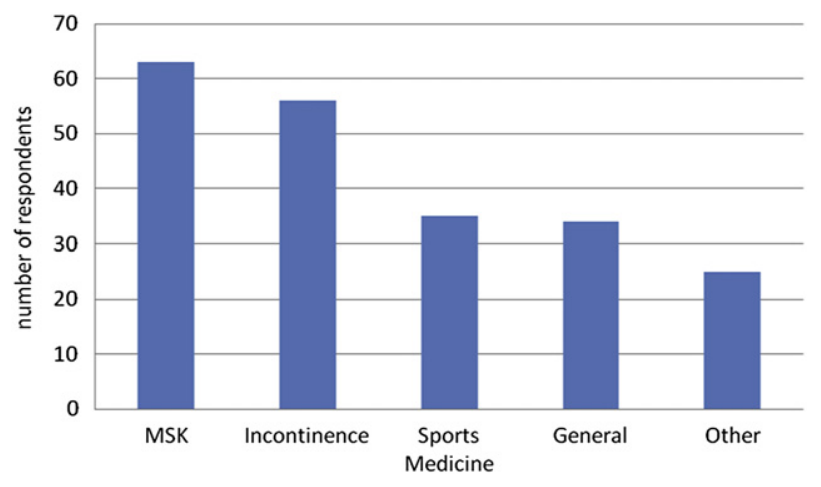

Figure 1. Question: What area/s of physiotherapy do you work in? 


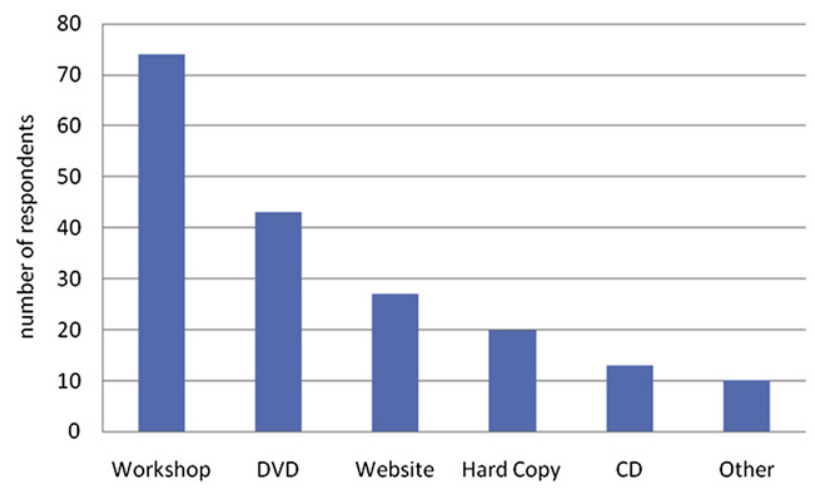

Figure 2. Question: What methods of training in diagnostic ultrasound would best suit you?

An open question was asked on the procedures that the physiotherapists perform with diagnostic ultrasound. The most common responses were for biofeedback in the areas of transversus abdominis, multifidus and the pelvic floor. Some musculoskeletal imaging was mentioned in the areas of the shoulders, hip muscles and elbow. Also mentioned was calculation of bladder volume.

\section{Training needs}

In relation to preferred method for training in ultrasound, the majority of respondents preferred that training be delivered via a workshop or DVD (see Fig. 2). Other suggestions included "an atlas to use with the machine" and "regional modules". Many respondents suggested the need for multiple forms of training and stressed the need to be able to ask questions.

Respondents mostly wanted more information about imaging anatomy and the use of machine controls (see Fig. 3). There was also a perceived need for training to cover things such as: "standards of practice for physiotherapists", "training standards" and "practical tutorials with patients". Some wanted "everything" covered and others felt no need for training.

The procedures respondents would most like training for are shown in Fig. 4. The most popular was the pelvic floor, followed closely by the abdominal muscles and shoulder. Other areas of interest for respondents were stated to be: "transperineal scanning of the pelvic floor", "all musculoskeletal areas", "measurement of muscle activity, companies need new measurement software for

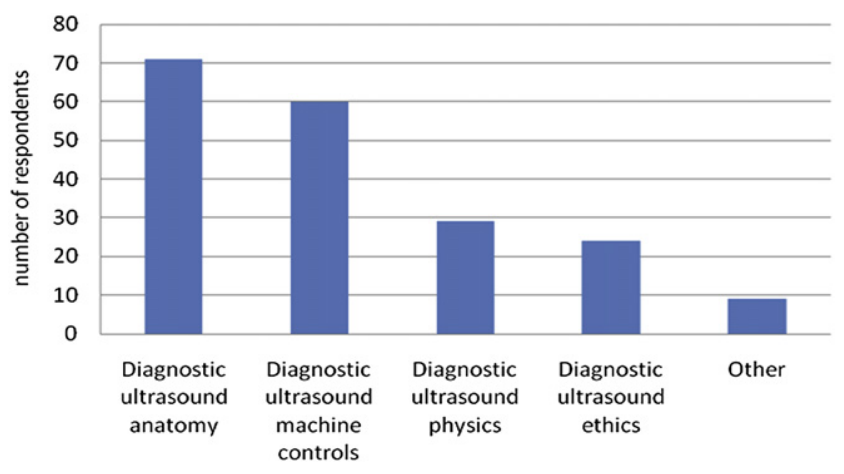

Figure 3. Question: What do you feel you need more information about?

physiotherapists to use in rehabilitation", "knee", "acute muscle tears", and "lymphoedema".

\section{Discussion}

This study explored current utilisation of diagnostic ultrasound imaging by physiotherapists. While there appears to be a keen interest in the modality by members of the profession, not all have access to a machine. Some physiotherapy practices are purchasing the machines and some individual physiotherapists are also purchasing equipment, which is interesting since the cost of the machines is relatively high and also all respondents report not using the machine a great deal during their daily workload.

Some physiotherapists are acquiring superseded machines from local radiology departments. While this may be good if the machines are still functioning well and have been well maintained, calibrated and serviced; several concerns are raised in relation to the condition of the equipment if it has been sitting in storerooms for a long period of time. Such equipment will also have higher specifications and additional capabilities, such as Doppler which is normally included on such machines. Doppler ultrasound involves higher intensities and power than that used with B mode imaging. ${ }^{15,16}$ As the survey showed, very few physiotherapists are directly purchasing equipment with Doppler capabilities therefore physiotherapists acquiring these superseded machines need additional education in this application and advice on how and when to use it since such machines have a greater potential for misuse if physiotherapists were to experiment with the Doppler capabilities.

Superseded machines also have a greater range of transducers, far beyond the requirements of physiotherapists. These include

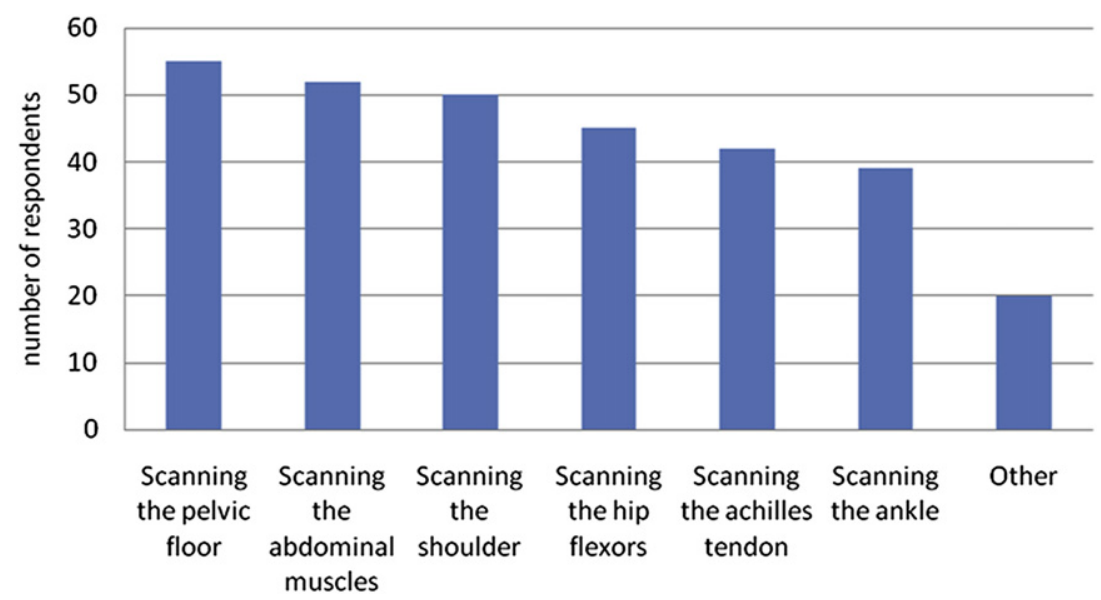

Figure 4. Question: What diagnostic ultrasound procedures would you most like to be trained in? 
endocavity transducers which raise ethical and legal issues of some concern. Radiology practices who pass superseded machines to physiotherapists potentially should also be offering the physiotherapists training in the equipment they are passing on. Both parties should be looking to establishing professional links to provide physiotherapists with support from their local experts who can help with training and guidance.

As many of the survey participants indicated they are currently considering the purchase of a machine, the experts now need to be thinking of developing relevant and accessible training, in readiness. The longer the physiotherapists go without expert training, the greater the potential for misuse of the machine and the possibility of inaccurate diagnoses and poor patient outcomes. The physiotherapist is also more likely to become frustrated and disillusioned with the modality. Part of any proposed training should include education on transducer selection.

Consideration should also be given to the scope of physiotherapy practice with diagnostic ultrasound. The physiotherapy professional body should be involved and develop guidelines and codes of practice in relation to physiotherapy use of the modality. The professional body should be encouraged to seek help, guidance and advice from the experts in the field when developing such guidelines. Such guidelines would help physiotherapists work through the medico legal implications of use of the modality within their profession. To avoid misdiagnosis and provide both therapist and patient with confidence in the use of the modality, guidelines for proof of training and competence levels should be considered.

A positive outcome of the survey was that a large proportion of respondents reported receiving at least some form of training in diagnostic ultrasound, even though the training may have been brief. However, to find that $32 \%$ are using the modality without any training is disturbing. Given the potential for misuse of the machine, the possibility of poor outcomes for patients and frustration for the physiotherapists, who may not be getting desired outcomes with the equipment, training is required. Consideration should also be given to the possibility of misdiagnosis and inappropriate or incorrect biofeedback to both patient and therapist.

Diagnostic ultrasound machine suppliers seem to recognise the need to provide some form of training when selling the equipment to physiotherapists. Suppliers should be encouraged to access training programs as they are developed or encourage links with local radiology departments when selling the equipment. Since radiology departments were mentioned in the survey as providing training, this supports the concept that the experts in the field are and should be providing the training, guidance and support network related to use of diagnostic ultrasound.

There is obvious interest in the modality within the physiotherapy profession and a large proportion of respondents have or are contemplating the purchase of equipment. However, response from respondents already in possession of machines indicated the majority were only using the machine $1-2$ times a day. Perhaps with training and a greater understanding of the role of the modality within clinical physiotherapy practice, it has the potential to be used more and become a more beneficial tool which could be used routinely by the physiotherapist.

Interestingly, while the majority of respondents indicated they worked in musculoskeletal physiotherapy, the procedures most commonly performed were assessment of the abdominal muscles and pelvic floor which would normally be the role of an incontinence or general physiotherapist. This raises the question of why is it not being used more clinically in musculoskeletal physiotherapy. It may be that physiotherapists regard these areas as more complex, perhaps they have not purchased the correct equipment or perhaps there is no available training specific to this clinical area.
In regards to preferred training method and establishment of training tools, the majority of respondents preferred face to face training. They reported feeling the need to have someone to discuss issues with, to be able to answer questions and actually show them how to scan and use the equipment. They preferred interactive training which could be achieved by either a workshop or DVD. They stated the need to start with the basics, including imaging anatomy and the use of machine controls. They reported needing help related to how to begin scanning and how to interpret the images. Once the basics have been mastered, scanning techniques might be expanded to explore structures in greater detail. It is commendable that physiotherapists recognise their need for such basic training. It was also interesting that the word "standards" appeared several times within open ended sentence responses, which indicates that physiotherapists recognise the need for some professional boundaries and guidelines in relation to their clinical practice and training. This would indicate that physiotherapists in the main are seeking help and guidance in the form of further training in diagnostic ultrasound.

Jedrzejczak and Chipchase ${ }^{17}$ conducted a study on the availability and usage of diagnostic ultrasound by physiotherapists in South Australia. This study found some similar results to this current survey. They reported that diagnostic ultrasound was being used mostly for biofeedback of the abdominal muscles, multifidus and pelvic floor. Of their respondents, all that were using the modality had received training and this training had been for $2 \mathrm{~h}$ or less. Respondents using diagnostic ultrasound were using it five times or less per week. It is interesting to see that with the passing of time, very little has changed in these areas. The study concludes by stating that training is necessary to ensure the safe and effective use of diagnostic ultrasound within physiotherapy.

\section{Limitations}

Limitations of this survey are related to the sampling method and response rate. Access to respondents was confined to physiotherapists registered on two existing databases. Potential respondents were invited to participate by an unsolicited e-mail and "overall response rates for e-mail surveys are known to be somewhat lower." 18 Also affecting the response rate is the fact that not all physiotherapists sent an e-mail would have an interest in diagnostic ultrasound and therefore would not be interested in completing the survey. The overall response rate was considered adequate for the purpose of the analysis and trends were apparent.

\section{Conclusion}

The physiotherapists who responded to the survey indicated an interest in or use of diagnostic ultrasound in their practice. Many currently, either have access to a machine or are contemplating purchase of a machine. The most common procedures performed were biofeedback in the areas of transversus abdominis, multifidus and the pelvic floor. Some musculoskeletal imaging was mentioned in the areas of the shoulders, hip muscles and elbow. They do not appear however, to be maximising the use of the modality during their daily workload. While most respondents indicated they had been provided with some form of training, most however, reported the need for further training, either in the form of a workshop or DVD. They prefer this training to concentrate on imaging anatomy, the use of machine controls and scanning the pelvic floor, abdominal muscles and shoulder. It is clear from the responses that diagnostic ultrasound is being used within physiotherapy. Training to optimise physiotherapists' clinical use of the modality is considered necessary and clearly desired by physiotherapists. 


\section{References}

1. Abu-Zidan FM, Freeman P, Mandavia D. The first Australian workshop on bedside ultrasound in the emergency department. New Zealand Medical Journa 1999;112(1094):322-4.

2. Australasian Society for Ultrasound in Medicine. Policies and statements, www. asum.com.au; 2008 [accessed 08.12.08].

3. Whittaker JL, Teyhen DS, Elliott JM, et al. Rehabilitative ultrasound imaging: understanding the technology and its applications. Journal of Orthopaedic and Sports Physical Therapy 2007;37(8):434-49.

4. McKiernan S, Chiarelli P, Warren-Forward H. Diagnostic ultrasound use in physiotherapy, emergency medicine and anaesthesiology. Radiography 2010;16:154-9.

5. Baessler K, Schussler B, Burgio KL, Moore KH, Norton PA, Stanton SL. Pelvic floor re-education principles and practice. 2nd ed. London: Springer; 2008.

6. Teyhen DS, Miltenberger CE, Deiters HM, et al. The use of ultrasound imaging of the abdominal drawing - in maneuver in subjects with low back pain. Journal of Orthopaedic and Sports Physical Therapy 2005;35(6):346-55.

7. Frost N, Clarke J. Ultrasound for biofeedback in physiotherapy. Sound Effects 2004; 4:10-3.

8. Pressler JF, Heiss DG, Buford JA, Chidley JV. Between-day repeatability and symmetry of multifidus cross-sectional area measured using ultrasound imaging. Journal of Orthopaedic and Sports Physical Therapy 2006;36(1): $10-8$.
9. Van K, Hides JA, Richardson CA. The use of real-time ultrasound imaging for biofeedback of lumbar multifidus muscle contraction in healthy subjects. Journal of Orthopaedic and Sports Physical Therapy 2006;36(12):920-5.

10. Dietz HP, Jarvis SK, Vancaillie TG. The assessment of levator muscle strength: a validation of three ultrasound techniques. International Urogynecology Journal 2002;13:156-9.

11. Whittaker JL, Thompson JA, Teyhen DS, Hodges P. Rehabilitative ultrasound imaging of pelvic floor muscle function. Journal of Orthopaedic and Sports Physical Therapy 2007;37(8):487-98.

12. Thompson JA, O'Sullivan PB, Briffa K, Neumann P, Court S. Assessment of pelvic floor movement using transabdominal and transperineal ultrasound. International Urogynecology Journal 2005;16:285-92.

13. Lydeard S. The questionnaire as a research tool. Family Practice 1991;8(1):84-91.

14. Polgar S, Thomas SA. Introduction to research in the health sciences. 4th ed. Edinburgh: Churchill Livingstone; 2000.

15. Gent R. Applied physics and technology of diagnostic ultrasound. South Australia: Milner Publishing; 1997.

16. Hoskins PR, Thrush A, Martin K, Whittingham TA. Diagnostic ultrasound physics and equipment. London: Greenwich Medical Media Limited; 2003.

17. Jedrzejczak A, Chipchase LS. The availability and usage frequency of real time ultrasound by physiotherapists in South Australia: an observational study. Physiotherapy Research International 2008;13(4):231-40.

18. Yun GW, Trumbo CW. Comparative response to a survey executed by post e-mail and web form. Journal of Computer-Mediated Communication 2000;6(1). 